\title{
Research on Location Problem of Multi-distribution Center based on Chaos Adaptive Mutation Particle Swarm Optimization Algorithm
}

\author{
Tiaotiao $\mathrm{Du}^{1}$, Kaijun $\mathrm{Wu}^{1}$, Tiejun $\mathrm{Wang}^{2}$ \\ ${ }^{1}$ School of Electronic and Information Engineering, LanZhou Jiao Tong \\ University, LanZhou 730070, China, \\ ${ }^{2}$ School of mathematics and computer science institute, Northwest University for \\ Nationalities, LanZhou 730030, China \\ E-mail:dutiaotiao@sina.com
}

\begin{abstract}
Location problem of multi-distribution center is a kind of NP hard problem. To solve such problems, this paper proposes a chaos adaptive mutation particle swarm optimization algorithm. The algorithm uses the ergodic property of chaos to initialize the particle swarm to enhance the diversity of the population, according to the variance of population fitness to adjust the probability of mutation, and adjust the inertia weight factor to improve the global and local search capability of the whole population. In this paper, the algorithm is applied to the location problem of multi-distribution center, established the multi-factor constraints of mathematical model which aiming at timeliness, and on this basis, the corresponding algorithm is designed. It can be seen from the location instance simulation results that the optimization results and efficiency of the adaptive mutation particle swarm optimization algorithm is better than the genetic algorithm and the standard particle swarm optimization algorithm.
\end{abstract}

Keywords: Distribution center location; Particle swarm optimization algorithm; Chaos adaptive mutation

\section{Introduction}

With the development of information technology, the economic gradually internationalize, the borderless business trends are becoming evident, new product market competition is becoming increasingly fierce, progress of technology and diversification of demand lead to ever-shortening product life cycle. Then the enterprise need shorter delivery, improve product quality, reduce costs and provide better service. In order to adapt to the environment, market competition between enterprises and enterprises has raised to the competition between the supply chains. At the same time the enterprise (supply chain) in order to be able to survive in the competition, there has been modern logistics industry which provided the basic impetus for the sustainable development of the national economy in high starting point.

Distribution is the core content of modern logistics, a connecting link between producers and consumers, and occupied a pivotal position in the modern logistics activities, what's more the distribution center as an important part of logistics system, plays an important role in the whole logistics system. The location of distribution center will directly affect the efficiency, cost and service of the supply chain, Scientific and reasonable distribution center location, can effectively conserve resources, improve the efficiency of the logistics system, enable mutual coordination and cooperation between supplier and customer demand point, reduce resource waste in the logistics system and provide powerful guarantee for the development of highly efficient logistics system 
balance. Weber[1] proposed the theory of location for the first time, mainly studied how to choose the location of a warehouse, so that it can reached the shortest distance to the scattered demand point. In 1964, Hakimi[2] made the site selection problem more theoretical, which led to the great concern of scholars, he proposed a network of multi -facility location. From then on, the site selection problem has become a systematic theory. With the continuous development of logistics industry, many experts and scholars are paying more attention to distribution center location problem. In the distribution center location model, mainly including the continuous location model [3]and network location model [4]and mixed integer programming model[5]. In the aspect of solving model, mainly involving branch and bound method[6],Lagrange relaxation method[7]and so on. With the development of intelligent optimization algorithm, a new method is proposed to solve the problem of continuous location of distribution center. Simulated annealing algorithm [8-9]' genetic algorithm[10-11], ant colony algorithm [12-13], differential evolution[14]algorithms obtained a preliminary application in solving this kind of problem, but the solution accuracy and solving efficiency, etc. should be improved.

Particle swarm optimization (PSO) is a kind of bionic algorithm which has recently appeared to mimic birds foraging[15],with the advantages of few individual numbers, simple calculation, fast convergence speed and easy implementation, and has been applied in the vehicle routing optimization problem[16-19], and achieved good results. However, due to the particle swarm algorithm is easy to stagnation, easy to fall into local optimum problems, especially in the case of numerous parameters and more local extreme points, the performance of optimizing would drop dramatically[20].A new chaos adaptive mutation particle swarm optimization algorithm is proposed in this paper, which use the ergodicity of chaos to initialize the particle swarm to enhance the diversity of the population, adjust the size of the mutation probability according to group fitness variance, and through adjusting inertia weight factor to improve the global and local search ability of the entire group. In this paper, the chaos adaptive mutation particle swarm optimization algorithm is applied to the distribution center location problem, by location instance simulation it can be seen that the chaos adaptive mutation particle swarm optimization algorithm achieved good effect in reducing the computation time and avoid prematurity, etc. and more superiority than other algorithm, also can find the lower cost.

\section{Mathematical Model of Multi-distribution Center Location}

\subsection{Problem Description}

In this paper, the distribution center location problem can be described as in the $\mathrm{m}$ counts alternative sites choose the $\mathrm{k}$ counts locations as a distribution center, distribution items for the $\mathrm{n}$ counts demand point, makes the transportation cost between selected distribution center and its distribution within the scope of the demand points is minimal.

\subsection{Basic Assumptions}

In order to facilitate solve the model, meanwhile the model is not too complicated and has practical value, make the following assumptions:(1)Select a new distribution center only within a certain range of alternative locations;(2)System only considers the transportation from the distribution center to the demand point;(3)Only consider the distribution of a product, and one-off complete the transportation of the distribute goods;(4)Transportation cost is proportional to the traffic and transportation distance;(5)Unit transportation rate between distribution center and each points of each user's is known as a constant;(6)User's demand for the product is known as a constant;(7) The fixed investment cost of establishing and managing the distribution center is known;(8)Capacity of distribution center is limited. 


\subsection{Symbol Definition}

$i$ is the serial number of demand points; $j$ is the serial number of distribution center; $k$ is the number of distribution center in preparation; $m$ is the number of alternative addresses for distribution centers; $n$ is the number of demand points; $d_{i j}$ is the distance between distribution center $j$ and demand point $i ; c_{i j}$ is the unit freight between distribution center $j$ and demand point $i ; q_{i j}$ is the transport volumes between distribution center $j$ and demand point $i ; s_{j}$ is the maximum capacity of distribution center $j ; p_{i}$ is the requirement of demand point $i ; r_{j}$ is the integer variable between 0 and $1 ; r_{j}=1$ represents the distribution center $j$ is selected; $r_{j}=0$ represents not selected; $x_{i j}$ is the integer variable between 0 and 1 which indicates whether the selected distribution center $j$ deliver goods to demand point $i ; v_{i j}$ is the average vehicle speed between distribution center $j$ and demand point $i ; T$ is the maximum allowable delivery time of demand point $i ; F_{j}$ is the fixed costs of alternative distribution center $j$,including basic investment fees and fixed operating expenses.

\subsection{Model Building}

The model established by objective function is shown in the formula (1).

$$
\min F=\sum_{i=1}^{n} c_{i j} d_{i j} q_{i j} x_{i j}+\sum_{j=1}^{m} r_{j} F_{j}
$$

Constraint conditions is as the formula (2) to formula (11) follows:

$$
k=\sum_{i=1}^{m} r_{j}
$$

$$
\sum_{i=1}^{n} q_{i j} \leq s_{j} r_{j} j=1,2, \ldots, m
$$

$$
\sum_{j=1}^{m} r_{j} \leq m \quad j=1,2, \ldots, m
$$

$$
\sum_{j=1}^{m} q_{i j} \geq p_{i} i=1,2, \ldots, n
$$

$$
T_{i j}=d_{i j} / v_{i j}, i=1,2, \ldots, n, j=1,2, \ldots, m
$$

$T_{i j} r_{j} x_{i j} \leq T_{i}, i=1,2, \ldots, n, j=1,2, \ldots, m$

$$
\sum_{j=1}^{m} x_{i j}=1 i=1,2, \ldots, n
$$


$q_{i j} \geq 0, \quad i=1,2, \ldots, n, j=1,2, \ldots, m$

(9)

$$
x_{i j} \in[0,1], i=1,2, \ldots, n, j=1,2, \ldots, m
$$

$$
r_{j} \in[0,1], j=1,2, \ldots, m
$$

Formula (1) represents the sum of transportation costs and fixed investment costs, its purpose is to find the minimum sum of the two costs; Formula (2) represents that the number of selected distribution centers is equal to the setting distribution centers; Formula (3) represents the capacity limit of distribution center; Formula (4) represents the number limit of distribution center; Formula (5) represents the supply constraint of distribution center; Formula (6) and formula (7) represents that the distribution time of each demand point must be within the allowable range to meet the timeliness requirements; Formula (8) represents that there is only one distribution center for supply at each demand point; Formula (9) represents that the transport volume is not a negative number of constraints; formula (10) and (11) are decision variables.

\section{Particle Swarm Optimization Algorithm}

\subsection{Standard PSO}

Particle swarm optimization algorithm is the intelligent group algorithm with a global search capability, which is initially developed from the simulation of social behavior [15].In the goal of an n-dimensional search space, the particle swarm is initialized distributed in space and the position of each particle spatial said a specific solution, each particle in the system to find the optimal position of the particle in the goal search space. Assuming that the number of particles in the system is initialized to $\mathrm{N}$, the position of the $i$ particle is represent by a vector of D-dimension, that is $X_{i}=\left(x_{i 1}, x_{i 2}, \ldots, x_{i D}\right)$, $i=1,2, \ldots, N, x_{i j}$ belongs to the scope of $\left[a_{j}, b_{j}\right]$, that is $a_{j} \leq x_{i j} \leq b_{j}, i=1,2, \ldots, N$, $j=1,2, \ldots, N$,Each position of the particle corresponds to a potential solution in the problem domain. The fitness value of the particle $X_{i}$ represents a potential solution to a specific problem, and different fitness values indicate the advantages and disadvantages of the solution. The corresponding velocity of a D-dimensional vector is expressed as $V_{i}=\left(v_{i 1}, v_{i 2}, \ldots, v_{i D}\right), p_{i}=\left(p_{i 1}, p_{i 2}, \ldots, p_{i D}\right)$ represents the searching optimal position of the particle so far, $p_{g}=\left(p_{g 1}, p_{g 2}, \ldots, p_{g D}\right)$ represents the searching optimal position of the particle so far. The update formula for the D-dimension velocity and position of the particle in the first $k+1$ iteration is shown in formula (12) and formula (13).

$$
v_{i d}^{k+1}=\omega v_{i d}^{k}+c_{1} r_{1}\left(p_{i d}^{k}-x_{i d}^{k}\right)+c_{2} r_{2}\left(p_{g d}^{k}-x_{i d}^{k}\right)
$$

$$
x_{i d}^{k+1}=x_{i d}^{k}+v_{i d}^{k+1}
$$

Among them, $w$ is known as the inertia weight, its size determines the number of particles in the current inheritable speed, used to balance the exploration and development capabilities of PSO. Larger w makes the particles in the original direction fly farther, has better ability of exploration, but slower convergence; smaller w makes the particles have better development ability, but it may lead to fall into local optimum. $c_{1}$ and $c_{2}$ are learning factors, which respectively represent the close degrees of the most advantage of 
the particle to their own history and the most advantage of the population. $r_{1}$ and $r_{2}$ are the random number of uniformly distributed in the $[0,1]$ region.

\subsection{Chaos Adaptive Mutation Particle Swarm Optimization Algorithm}

3.2.1. Chaos and its characteristics: Chaos is a common phenomenon in nonlinear systems, which is complex and random. The basic idea of Chaos optimization algorithm is to first produce a set number of chaotic variables with the same as optimization variables, enlarging the traversing range of chaotic motion to the range of optimization variables, and then directly using the properties of randomness and ergodicity to search. Since the chaotic search algorithm sensitivity to initial conditions, easy to jump out of local minima and fast search speed, search technology based on chaos is no doubt more superior than other search techniques.

In order to overcome the phenomenon of premature convergence and stagnation in the later period of the PSO algorithm, this paper will add the chaotic search of Logistic map to the PSO algorithm [21].

Logistic mapping expression as shown in formula (14):

$$
Z_{n+1}=4 Z_{n}\left(1-Z_{n}\right) 0 \leq Z_{0} \leq 1
$$

In the formula: $Z_{n}$ is the chaotic variable, $n=0,1,2, \ldots ; Z_{0}$ is the initial value of chaotic variables, its tiny difference in a long time will lead to a great difference, so the chaos can according to its own laws do not repeatedly traverse all states of the search space.

3.2.2. Chaos initialization strategy: Randomly generated a $\mathrm{D}$-dimension vector $z_{1}=\left(\mathrm{z}_{11}\right.$, $\left.z_{12}, \ldots, \mathrm{z}_{1 Q}\right)$, each component in the vector in the $[0,1]$ region, according to formula (14) can get $z_{1}, z_{2}, \ldots, z_{N}$, map all the components of $z_{i}$ to the range of the optimization variables: $\left.x_{i j}=a_{j}+\left(b_{j}-a_{j}\right) z_{i j} i=1,2, \ldots, N ; j=1,2, \ldots, Q\right)$. By calculating the objective function, the $m$ solution with better performance is selected from the initial population of $N$ as the initial solution of the optimization problem, and randomly generated the initial velocity of $m$.

3.2.3. Community fitness variance: Because the position of each particle can be reflected through the fitness function value. So by setting all particles in the population fitness function value changes can realize the descriptions of each particle aggregation degree. Here, introduce the concept of community fitness variance.

The total number of particle swarm is $n$, The fitness function of the $i$ particle is $f_{i}$, $f_{\text {avg }}$ is the average fitness value of particle swarm, then the definition of population fitness variance is shown as the formula (15):

$$
\sigma^{2}=\sum_{i=1}^{n}\left(\frac{f_{i}-f_{\text {avg }}}{f}\right)^{2}
$$

In the formula, the purpose of $f$ is to limit the size of $\sigma^{2}$, and its value varies with the change of the algorithm, can be calculated by formula (16).

$$
f=\max \left\{1, \max \left\{\left|f_{i}-f_{\text {avg }}\right|\right\}\right.
$$

The variance of population fitness reflects the degree of convergence of all the particles in the particle swarm, and the smaller the value, the more the particle swarm tends to converge; otherwise, the particle swarm is in a random search phase. 
3.2.4. Adaptive mutation operation: In order to make the particles search in a new direction when the algorithm is trapped in the search, so the introduction of adaptive mutation should be based on the degree of aggregation of the group. That is to say, the variation of the probability changes with the change of $\sigma^{2}$. In this paper, the variation probability is calculated by the formula (17).

$$
p_{m}=\left(p_{\text {max }}-p_{\text {min }}\right)\left(\sigma_{m}^{2} / n\right)^{2}+\left(p_{\text {min }}-p_{\text {max }}\right)\left(\sigma_{m}^{2} / n\right)+p_{\text {max }}
$$

In the formula, $p_{m}$ represents the mutation probability of all groups extreme values in the $m$ iteration; $\sigma_{m}^{2}$ is the community fitness variance in the $m$ iteration; $p_{\max }$ is the maximum value of the current mutation probability, $p_{\min }$ is the minimum value of the current mutation probability.

From the formula (15) it is easy to see that, when the fitness variance is smaller, the greater global extreme value of the mutation probability; on the contrary, the smaller global extreme value of the mutation probability. The algorithm can adjust the mutation probability adaptively according to the position of the particle in the group, achieve the goal of jump out of local optimal solution.

For the mutation operation of $g b$, the method of increasing disturbance is adopted, set $g b^{k}$ is the $k$ iteration value of $g b, \eta$ obeys Gauss $(0,1)$ distribution, then the mutation formula of $g b^{k+1}$ is shown as the formula (18).

$$
g b^{k+1}=g b^{k}(1+0.5 \eta)
$$

3.2.5. Adjustment of inertia weight factor $\omega$ : The inertia weight factor is the coefficient of the original velocity. When the value is large, it is advantageous to search a new region. When the value is small, it is helpful for the particle to search carefully in the current area. In this paper, the inertia weight factor used the decreasing strategy to achieve the goal of optimization, and the formula is shown in formula(19):

$$
\omega_{i}=\omega_{\max }-\left(\omega_{\max }-\omega_{\min }\right)\left(i / I_{\max }\right)
$$

Among them, $\omega_{i}$ is the inertia weight factor in the $i$ iteration, $I_{\max }$ is the maximum number of iterations, $\omega_{\min }$ and $\omega_{\max }$ respectively represent the setting minimum and maximum inertia weight factor.

\section{Particle Swarm Optimization Algorithm for Multi-distribution Center Location Problem}

\subsection{Particle Coding Design}

The key problem of particle swarm optimization algorithm is that the position of the particle is corresponding to the solution of the problem. In this paper, we use the method of literature [22] to construct the coding strategy of the particle swarm. In the coding of particles, the dimension of the particles is the number of demand points, $i=\left(w_{1}, w_{2} \ldots\right.$, $w_{n}$ ), the value of each dimension in turn is the ordinal number of the demand point, That is, the $\mathrm{K}$ dimensional representation of the $\mathrm{k}$ demand point; Each dimension of the particle is valued, and its value is the ordinal number of any alternative distribution center, for example, $m=6, n=10$, thus $\operatorname{dos}[\mathrm{i}]=m(m=1,2, \ldots, 6)$. Because each demand point in this article can have a distribution center for the supply, so each particle on each 
dimension corresponds to an integer value, the value of this represent which distribution center is selected. Coding schematic diagram is shown in table 1. 
Table 1. Encoding Schematic

\begin{tabular}{cllllllllll}
\hline $\operatorname{dos}[i]$ & 2 & 4 & 3 & 1 & 1 & 2 & 3 & 6 & 4 & 1 \\
\hline$i$ & 1 & 2 & 3 & 4 & 5 & 6 & 7 & 8 & 9 & 10 \\
\hline
\end{tabular}

The above coding indicates that the demand point 1 is supplied by the distribution center 2 ; the demand point 2 is supplied by the distribution center 4 ; the demand point 3 is supplied by the distribution center 3; the demand points 4 and 5 are supplied by the distribution center $1 ; \ldots . .$. the demand point 10 is supplied by the distribution center 1 .

Task allocation of algorithm is realized by a two-dimensional array, the two-dimensional array will sequentially deposit the number of distribution center by line, each column of the row deposit the demand point to be supplied. From the above example of coding can get the corresponding two-dimensional array, as shown in figure 1, the demand points 4,5 and 10 are supplied by the selected distribution center 1 ; the demand points 1 and 6 are supplied by the selected distribution center $2 ; \ldots \ldots$; the demand point 8 is supplied by the selected distribution center 6 .

$$
\left[\begin{array}{l}
1 \\
2 \\
3 \\
4 \\
6
\end{array}\right] \Rightarrow\left\{\begin{array}{lll}
4 & 5 & 10 \\
1 & 6 & \\
3 & 7 & \\
2 & 9 & \\
8 & &
\end{array}\right\}
$$

\section{Figure 1. Schematic Diagram of Two-dimensional Array Corresponding to Coding}

\subsection{Algorithm Implementation Process}

Step 1: Parameter initialization. Input the number of particle swarm $n$,inertia weight factor $\omega_{\min }$ and $\omega_{\max }$, learning factor $c_{1}$ and $c_{2}$, mutation probability $p_{\max }$ and $p_{\min }$, minimum group variance $\sigma_{\min }$ and maximum iteration $I_{\max }$.

Step 2: According to 2.2.2 strategy to the particle's position and initialize the velocity at random.

Step 3: Fitness evaluation.Treat formula (1) as a fitness function to calculate the fitness value of each particle, namely total distance of empty vehicle, and check to see if it satisfies (2) to (4) constraints, if not, make $F=F_{\text {max }}, F_{\text {max }}$ is a large number, search again.

Step 4: For each particle in the swarm, the position and velocity of each particle are updated according to the formula (5) and the formula (6).Calculate fitness value of all particles, compared the fitness value of each particle with the best position $p b$ of the particle current passing, and then choose the optimal individual extremum. For each particle, compared the fitness value with the current best position $g b$ of all particles, and then select the global optimal extremum.

Step 5: Calculate the fitness variance $\sigma^{2}$ of groups according to the formula (8)

Step 6: Dynamically set the inertia weight factor $\omega$ according to the formula (12).

Step 7: Calculate the mutation probability $p_{m}$ according to the formula (10). 
Step 8: Generate a random number $r \in[0,1]$, if $r<p_{m}$, carry on the mutation operation by the formula (11),otherwise transferred to Step9.

Step 9: Chaotic optimization the global optimal extremum $g b$.First, the global optimal extremum is mapped to the definition domain of the equation $[0,1]$, and then iterate by the formula (7) to generate $\mathrm{N}$ series of chaotic variable, get $\mathrm{N}$ numbers particles, calculate the fitness function value of each particle, and get the optimal solution $g b^{\prime}$.

Step 10: Replace the position of the particle in the current population with $g b^{\prime}$.

Step 11: Determine whether the current number of iterations reaches the maximum number of settings, if achieved, then stop the iteration, output of the optimal solution $g b$, otherwise transferred to Step3.

\section{Example Simulation}

Suppose there are 30 demand, alternative distribution center are the first 10 demand points, the maximum capacity of each center are 100 tons, select three points as distribution center, the position coordinates (unit: $\mathrm{km}$ ) and the requirements of demand point(unit: $\mathrm{km}$ ) as shown in Table 2. the other parameters are set as follows: unit freight for 30 yuan / km - tons, each point of the construction costs are 20000 yuan, the average speed of each demand point vehicle is $25 \mathrm{~km} / \mathrm{h}$, the maximum allow delivery time of distribution is 2 hours.

Table 2. Coordinates and Requirements of Demand Point

\begin{tabular}{|c|c|c|c|}
\hline Client & $\begin{array}{l}\text { Horizontal } \\
\text { coordinate }\end{array}$ & $\begin{array}{l}\text { Vertical } \\
\text { coordinate }\end{array}$ & Demands \\
\hline 1 & 24 & 6 & 7 \\
\hline 2 & 32 & 78 & 13 \\
\hline 3 & 98 & 87 & 3 \\
\hline 4 & 15 & 95 & 15 \\
\hline 5 & 82 & 55 & 9 \\
\hline 6 & 99 & 36 & 12 \\
\hline 7 & 63 & 80 & 5 \\
\hline 8 & 43 & 27 & 12 \\
\hline 9 & 94 & 70 & 6 \\
\hline 10 & 67 & 23 & 13 \\
\hline 11 & 47 & 86 & 16 \\
\hline 12 & 52 & 32 & 9 \\
\hline 13 & 81 & 32 & 7 \\
\hline 14 & 27 & 54 & 6 \\
\hline 15 & 76 & 45 & 8 \\
\hline 16 & 18 & 39 & 14 \\
\hline 17 & 3 & 85 & 6 \\
\hline 18 & 74 & 3 & 3 \\
\hline 19 & 67 & 52 & 8 \\
\hline 20 & 28 & 94 & 4 \\
\hline 21 & 36 & 48 & 16 \\
\hline 22 & 81 & 71 & 5 \\
\hline 23 & 7 & 22 & 9 \\
\hline
\end{tabular}




\begin{tabular}{lccc}
24 & 46 & 100 & 11 \\
25 & 55 & 44 & 15 \\
26 & 6 & 60 & 14 \\
27 & 95 & 50 & 9 \\
28 & 46 & 5 & 12 \\
29 & 47 & 68 & 8 \\
30 & 94 & 25 & 6 \\
\hline
\end{tabular}

Respectively using genetic algorithm, particle swarm optimization algorithm and chaos adaptive mutation particle swarm optimization algorithm to calculate the example on the same computer, optimal scheme of three algorithms for the calculation as shown in table 3 and figure 2, the curve of the objective function changes with the number of iterations are shown in figure 3. It is easy to see from the calculated results that the chaos adaptive mutation particle swarm optimization algorithm can be quickly obtained the optimal solution with the multiple vehicles routing problem and time window, and the searching time and efficiency of optimal solution were better than the standard genetic algorithm and particle swarm optimization algorithm, providing a new method for solving multi-distribution center location problem.

Table 3. Location Scheme

\begin{tabular}{|c|c|c|}
\hline $\begin{array}{l}\text { Selection of } \\
\text { distribution centers }\end{array}$ & Distribution range & Cost \\
\hline 2 & $4,11,14,17,20,24,26,29$ & 76955 \\
\hline 5 & $3,6,7,9,13,15,19,22,27,30$ & 63851 \\
\hline 8 & $1,10,12,16,18,21,23,25,28$ & 91187 \\
\hline Total & & 231993 \\
\hline
\end{tabular}

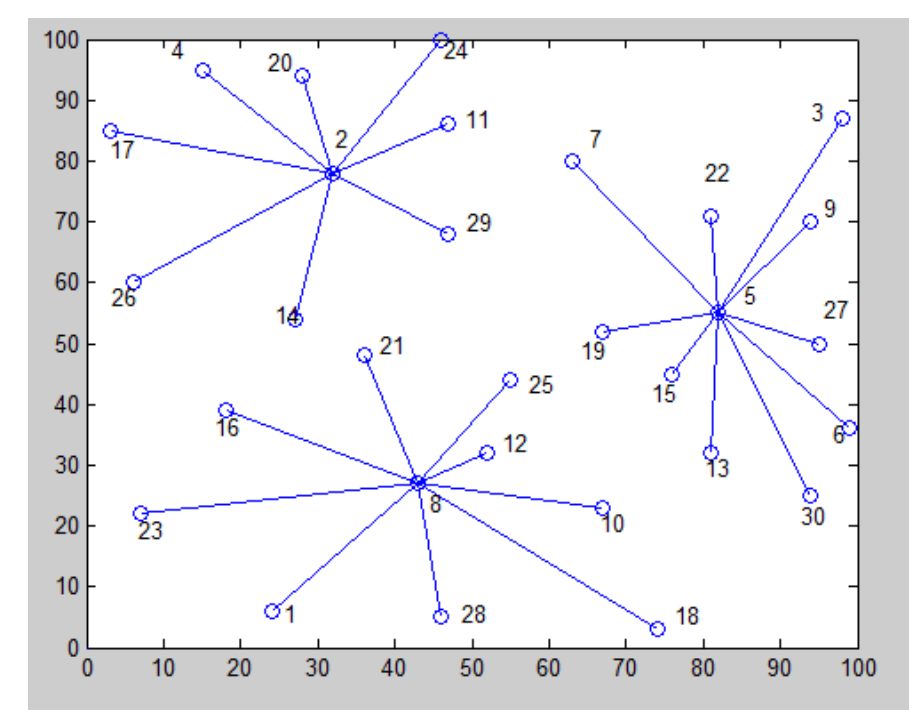

Figure 2. Optimal Addressing Scheme 


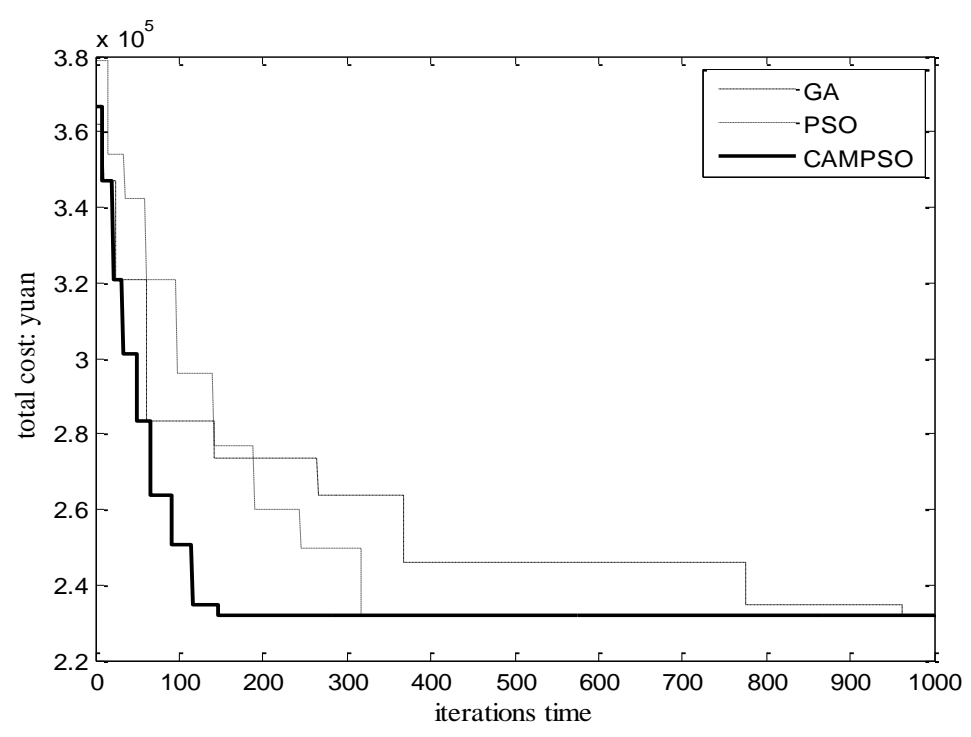

Figure 3. Contrast Diagram of Optimization Curve

\section{Conclusions}

This paper proposed a new chaos adaptive mutation particle swarm optimization algorithm , the algorithm using the ergodicity of chaos to initialize the particle swarm to enhance the diversity of the population, adjust the size of the mutation probability according to group fitness variance, and by adjusting inertia weight factor to improve whole groups of global and local search ability. Finally, the adaptive mutation particle swarm optimization algorithm is applied to solve the multi-distribution center location model. The simulation results show that the adaptive mutation particle swarm optimization algorithm is more effective in solving the distribution center location problem. However, the parameter setting of the adaptive mutation particle swarm optimization algorithm and its theoretical proof are still in the exploration, which still need to be further studied. In addition, the distribution center location model is still in an ideal stage, many practical factors are not considered, therefore, how to make the model closer to the actual is an important direction for the future research.

\section{Acknowledgements}

This work was supported by the National Social Science Foundation of China (No. 15CGL001)

\section{References}

[1] A. Weber, "Alfred Weber's Theory of the Location of Industries", tyofChicago, (1929).

[2] S. L. Hakimi, "Optimal location of switching centers and the absolute centers and medians of a graph", Operations Research, vol. 12, no. 3, (1964), pp. 450-459.

[3] B. Goldengorin, D. Ghosh and G. Sierksma, "Branch and peg algorithms for the simple plant location problem", Computers \& Operations Research, vol. 30, no.7, (2003), pp. 967-981.

[4] M. A. Efroymson and T. L. Ray, "A branch-and-bound algorithm for plant location", Operations Research, vol. 14, no. 5, (1996), pp. 361-368.

[5] S. M. S. Mapa and R. D. S. Lima, "Combining geographic information systems for transportation and mixed integer linear programming in facility location-allocation problems", Journal of Software Engineering \& Applications, vol. 7, no. 10, (2014), pp. 844-858.

[6] W. U. Ying and C. C. Sheng, "City distribution center:truck load matching based on branch and bound method", Journal of Changsha University of Science \& Technology, (2008), pp. 1405-1411.

[7] H. W. Cai and D. Y. Zhang, "Optimization on integrated production-location-routing problem based on lagrange relaxation algorithm", Chinese Journal of Management Science, vol. 18, no. 3, (2010), pp. 
53-57.

[8] S. M. Mousavi and R. Tavakkoli-Moghaddam, "A hybrid simulated annealing algorithm for location and routing scheduling problems with cross-docking in the supply chain”, Journal of Manufacturing Systems, vol. 32, no. 2, (2013), pp. 335-347.

[9] N. Avadian, R. Tavakkoli-Moghaddam, M. Amiri-Aref and S. Shiripour, "Two meta-heuristics for a multi-period minisum location-relocation problem with line restriction", International Journal of Advanced Manufacturing Technology, vol. 71, (2013), pp. 1-16.

[10] N. Lin and L. I. Zhi, "Study on location selection of logistics distribution center based on gis and genetic algorithm", Remote Sensing Information, vol. 32, no. 5, (2010), pp. 110-114.

[11] D. L. Jiang and X. L. Yang, "Genetic algorithm for continuous location problem of physical distribution center for decaying products", Systems Engineering-theory \& Practice, vol. 23, no. 2, (2003), pp. 62-67.

[12] Ibrahim and L. F, "Enhanced clustering techniques for hyper network planning using minimum spanning trees and ant-colony algorithm", Journal of Computer Science, vol. 7, no. 3, (2011), pp. 440-447.

[13] R. Musa, J. P. Arnaout and H. Jung, "Ant colony optimization algorithm to solve for the transportation", (2010).

[14] R. Pitakaso and T. Thongdee, "Solving a Multi-objective, Source and Stage Location-Allocation Problem Using Differential Evolution, Logistics Operations, Supply Chain Management and Sustainability", Springer International Publishing, (2014).

[15] J. Kennedy and R. Eberhart, "Particle swarm optimization", IEEE International Conference on Neural Networks, vol. 4, (1995), pp. 1942-1948.

[16] G. Qureshi, D. P. R. Bajaj and P. V. Puranik, "Particle swarm optimization with genetic operators for vehicle routing problem", International Journal of Engineering Science \& Technology, vol. 4, no.7, (2012).

[17] T. J. Wang and K. J. Wu, "Study on multi-depot vehicle routing problem based on cloud adaptive particle swarm optimization", Applied Mechanics \& Materials, (2012), pp. 253-255, 1369-1373.

[18] M. R. Khouadjia, B. Sarasola, E. Alba, L. Jourdan and E. G. Talbi, "A comparative study between dynamic adapted PSO and VNS for the vehicle routing problem with dynamic requests", Applied Soft Computing, vol. 12, no. 4, (2012), pp. 1426-1439.

[19] J. He, Y. Huang and W. Yan, "Yard crane scheduling in a container terminal for the trade-off between efficiency and energy consumption", Advanced Engineering Informatics, vol. 29, no. 1, (2015), pp. 59-75.

[20] W. U. Kaijuna and L. U. Huaiwei, "Model and algorithm of emergency logistics vehicle distribution based on time constraints", Computer application research, vol. 29, no. 8, (2012), pp. 2882-2885.

[21] Y. Shimizu, T. Miura and M. Ikeda, "A parallel computing scheme for large-scale logistics network optimization enhanced by discrete hybrid PSO", Computer Aided Chemical Engineering, vol. 27, no. 9, (2009), pp. 2031-2036.

[22] W. Zhao, "Study of multiple distribution center location based on particle swarm optimization", Journal of Wuhan University of Technology, vol. 36, no. 3, (2012), pp. 501-505. 